\title{
DESAFIOS DE SERVIDORES PÚBLICOS DO DISTRITO FEDERAL PARA TRANSFERENCIA DE TREINAMENTO NO TRABALHO: ANÁLISE QUALITATIVA COM USO DO IRAMUTEQ
}

\author{
Lana Montezano ${ }^{1}$, Pedro Resende Junior ${ }^{1}$, Ronaldo Santana ${ }^{2}$ e Luiz Fernando da Silva ${ }^{2}$ \\ ${ }^{1}$ Universidade de Brasília, Brasil. lanamontezano@gmail.com; pcrj73@gmail.com \\ 2 Universidade Coorporativa dos Correios, Brasil. ronaldo.sprint@hotmail.com; fernandocps@hotmail.com
}

\begin{abstract}
Resumo. A revisão de literatura utilizada aponta que o desenvolvimento de competências dos servidores provenientes de ações de treinamento possui relação direta com a melhoria do serviço público para a população. O artigo teve como objetivo descrever, sob a perspectiva da abordagem qualitativa, as variáveis inibidoras da transferência de treinamento no contexto específico do setor público do Distrito Federal do Brasil. A pesquisa obteve uma amostra de 1427 servidores públicos que passaram por treinamento num intervalo máximo de 12 meses até a coleta de dados. As respostas foram tratadas com o software Iramuteq. Os principais resultados apontam que foram encontradas três classes de conteúdo: (1) Dificuldade de Aplicação Prática (falta de aderência entre o treinamento praticado e a realidade do contexto do trabalho do servidor público); (2) Inconsciência da Dificuldade (falta de tempo para perceber as possíveis dificuldades de transferência de treinamento); e, (3) Resistência Cultural (falta de abertura à mudança), as quais rementem a dimensão de ambiente de trabalho como fator de restrição à transferência. Além disso, constatou-se que o uso de software qualitativo contribuiu para a geração dos resultados, facilitando o processo de análise devido ao tamanho da amostra obtida.
\end{abstract}

Palavras-chave: Treinamento; Transferência de Treinamento; Organizações Públicas; Método Qualitativo.

CHALLENGES OF PUBLIC EMPLOYEES IN THE FEDERAL DISTRICT TO TRANSFER TRAINING AT WORK: A QUALITATIVE ANALYSIS USING IRAMUTEQ

\begin{abstract}
The literature review used points out that the development of employee skills is directly related to the improvement of public service for the population. The article aimed to understand, from the perspective of the qualitative approach, the variables that inhibit the transfer of training in the specific context of the public sector of the Federal District of Brazil. The survey obtained a sample of 1427 public servants who underwent training in a maximum interval of 12 months until data collection. The answers were treated with the Iramuteq 0.7 . The main results indicate that three content classes were found: (1): Practical Application Difficulty (lack of adherence between the training practiced and the reality context of the public employee's work); (2) Unconsciousness of Difficulty (lack of time to understand possible difficulties in transferring training); and, (3) Cultural Resistance (lack of openness to change), which complement the dimension of the work environment as a restriction factor to transfer. In addition, it was found that the use of qualitative software contributed to the generation of results, facilitating the analysis process due to the size of the sample obtained.
\end{abstract}

Keywords: Training; Training Transfer; Public Organizations; Qualitative Method.

\section{INTRODUÇÃO}

As ações de treinamento aos servidores públicos visam a melhoria da gestão pública para oferta de serviços públicos adequados à sociedade (Ranzini \& Bryan, 2017). Rodrigues, Petinem \& Rodriges (2020) corroboram com tal afirmativa ao apresentar evidências que o 
desenvolvimento de competências dos servidores provenientes de ações de treinamento possui relação direta com a melhoria do serviço público para a população, as quais devem ser feitas com responsabilidade aos gastos públicos e alinhamento às diretrizes estratégicas da organização.

Uma das finalidades do treinamento é desenvolver competências nos participantes para que possam aplicá-las no trabalho (Ford, Baldwin \& Prasad, 2018; Hughes, Zajac, Spencer, \& Salas, 2018), ou seja, o principal objetivo do treinamento, em contextos organizacionais, é proporcionar a transferência positiva do treinamento (Baldwin, Ford, \& Blume, 2017). No entanto, apesar dos elevados investimentos em treinamentos, ainda há o desafio relacionado à sua transferência para o trabalho (Baldwin, Ford, \& Blume, 2017), de modo que as organizações possam ser beneficiadas, devido ao fato de que as competências treinadas não são aplicadas (Hughes et al. 2018).

Antunes, Nascimento, \& Bates (2018) reforçam a necessidade de conhecimento mais aprofundado acerca da transferência de treinamento, em função de que apenas parte das competências ensinadas serem transferidas ao trabalho. Neste sentido, conhecer as informações que influenciam na transferência de treinamento permitirá o aumento do rendimento dos investimentos em treinamentos realizados pelas organizações, uma vez que será possível planejar e executar as iniciativas adequadamente (Ford, Baldwin, \& Prasad, 2018). Inclusive Baldwin, Ford, \& Blume (2017) recomendam a compreensão do significado de transferência na percepção dos treinandos para que possam ser desenvolvidas melhores instrumentos de mensuração.

Blume, Ford, Baldwin \& Huang (2010) conduziram uma meta-análise sobre transferência de treinamento e identificaram que os principais fatores contextuais que afetam esta transferência são o clima organizacional, apoio e restrições situacionais no trabalho. Isso reforça a necessidade de identificação das dificuldades que os treinandos possuem para colocar a aprendizagem em prática, o que gerou a seguinte questão de pesquisa: "Quais as principais dificuldades que os servidores públicos brasileiros possuem para colocar em prática no seu trabalho o aprendizado adquirido no treinamento?"

Sendo assim, este artigo tem como objetivo descrever, sob a perspectiva da abordagem qualitativa, as variáveis inibidoras da transferência de treinamento no contexto específico do setor público do Distrito Federal do Brasil. Apesar dos diversos estudos e avanços já realizados na identificação de variáveis relacionadas à transferência (Ford, Baldwin \& 
Prasad, 2018), esta pesquisa contribui para compreensão no contexto específico de servidores públicos, a partir da identificação de restrições que precisam ser superadas para que haja mair efetivadade dos gastos públicos em investimentos com treinamentos dos profissionais, de modo que sejam adequadamente preparados e tenham condições de colocar em prática para oferta de serviços públicos de maior qualidade.

\section{REFERENCIAL TEÓRICO}

Uma das primeiras definições de transferência de treinamento foi proposta por Baldwin e Ford (1988), referindo-se à aplicação eficaz no trabalho do que foi aprendido durante o treinamento. A transferência pode ser positiva quanto facilita do desempenho no trabalho ou negativa quando dificulta. Em revisão da literatura da produção sobre treinamento durante 100 anos, Bell et al. (2017) identificaram preocupações de estudos em reduzir a transferência negativa para evitar a interferência do contexto de transferência ao trabalho. Como por exemplo, tem-se no estudo de Ellington, Surface, Blume e Wilson (2015) a identificação de que o intervalo de tempo maior da aprendizagem interfere negativamente na transferência ao trabalho.

A transferência de treinamento depende algumas váriáveis, como as particularidades dos diferentes objetivos e expectativas que cada indivíduo tem em relação ao processo de aprendizado, as quais vão mudando ao longo do tempo, além da dependência de que ocorra uma situação de oportunidade para que possa colocar em prática (Baldwin, Ford, \& Blume, 2017).

Ford, Baldwin e Prasad (2018) indicam que são necessárias condições para que o aprendiz possa colocar em prática no trabalho o aprendizado, as quais são decorrentes de três dimensões: características dos indivíduos (personalidade, competências, estados de aprendizagem, motivação, e autoeficácia), do planejamento e implematação do treinamento (estratégias de aprendizagem, demonstração, desenho, objetivos), ambiente do trabalho (suporte do gestor e dos colegas, oportunidade de execução).

Hughes et al. (2018) também abordam estas três dimensões relacionadas à transferência de treinamento, e reforçam quanto à necessidade de um sistema de suporte para transferência de treinamento, abarcando o suporte dos gestores e colegas, o estabelecimento de metas para utilização no trabalho do que foi aprendido, feedbacks de desempenho relacionado à 
aplicação, mensuração da transferência de treinamento, uso de incentivos e recompensas pelo uso do que foi aprendido.

Observa-se que diversos estudos apontam a relevância do suporte para transferência, sendo que algumas variáveis podem ter maior influência, conforme Blume et al. (2010) identificaram que o suporte dos gestores tem maior impacto na transferência de treinamento do que o suporte dos pares. Al-Swidi e Yahya (2017) também constataram que o apoio do supervisor é determinante para que ocorra a transferência, além do estilo de aprendizagem.

Antunes, Nascimento, e Bates (2018) indicam a necessidade de se elaborar políticas de treinamento considerando variáveis individuais e situacionais devida a influência delas no processo de transferência do treinamento. Para tanto, identificaram três dimensões que contribuiem para a aplicação do que foi aprendido: ambiente de suporte tanto de gestores quanto de colegas; a motivação para transferir; e a transferibilidade que envolve aspectos como a validade de conteúdo, o desenho de tranferência e a oportunidade de uso.

\section{METODOLOGIA}

\subsection{Caracterização geral da pesquisa}

Este artigo é caracterizado como teórico-empírico, de natureza descritiva, com recorte transversal, e abordagem qualitativa com uso de questionário. Considerando o objetivo da pesquisa, o público-alvo foram os servidores públicos do Distrito Federal do Brasil, sejam eles da Esfera Federal ou Distrital, e que tivessem participado de alguma ação de treinamento voltada para o trabalho até 12 meses antes da data de aplicação da pesquisa, a qual ocorreu no $1^{\circ}$ semestre de 2018.

Este artigo faz parte do escopo de uma pesquisa mais ampla, com abordagem qualitativa e quantitativa, que teve como finalidade compreender as variáveis que influenciam na transferência de treinamento no contexto específico do setor público, conforme recomenado por Ford, Baldwin \& Prasad (2018) e Antunes, Nascimento, \& Bates (2018), sendo que a delimitação de escopo desta pesquisa apresentará resultados do fenônemo citado via abordagem qualitativa. 


\subsection{Procedimentos de Coleta de Dados}

Foi desenvolvido um Termo de Consentimento Livre e Esclarecido (TCLE) para a execução do procedimento de coleta de dados. $O$ instrumento de pesquisa foi estruturado da seguinte forma: (i) a apresentação da pesquisa com delimitação do público-alvo que deveria preencher o formulário, o objetivo da pesquisa, aspectos de sigilo do uso de dados individuais, identificação dos responsáveis pela pesquisa para coleta via formulário eletrônico; (ii) questões de múltipla escolha sobre caracterização do treinamento que participaram nos últimos 12 meses, referenciando a data de preenchimento da pesquisa, para delimitação de uma experiência específica a ser considerada para responder as questões; (iii) Escala de Motivação para Transferir desenvolvida e validada por Abbad, Lacerda e Pilati (2012) a ser mensurada com escala tipo Likert de 5 pontos; (iV) questões de múltipla escolha sobre aplicação no trabalho do conteúdo aprendido e questões abertas sobre motivos para colocar em prática este conteúdo e as principais dificuldades para aplicação no trabalho; (v) caracterização sócio profissional do respondente.

A estratégia de coleta de dados utilizada foi realizar parceria com a Escola Nacional de Administração Pública (ENAP) para envio de e-mail com o link da pesquisa no formato do Google Forms aos servidores públicos que tivessem participado de alguma ação de treinamento ofertada pela ENAP de fevereiro de 2017 a fevereiro de 2018. A escolha desta parceira foi devido ao fato de ser o órgão da Administração Pública responsável por desenvolver competências nos servidores públicos para torná-los capazes de propciar inovações, alcance dos resultados e melhores serviços à sociedade. O link do questionárioo eletrônico ficou disponível para preenchimento de março a agosto de 2018.

\subsection{Amostra da pesquisa}

Obteve-se uma amostra de 1.427 participantes voluntários na pesquisa, totalizando um corpus emergiram 10.840 ocorrências (palavras, formas ou vocábulos), sendo 1.451 palavras distintas e 722 palavras com uma única ocorrência referente às respostas da questão sobre dificuldades para aplicação no trabalho do conteúdo aprendido.

O perfil desta amostra é caracterizado principalmente com $52 \%$ do sexo feminino; com idade média de 40 anos ( $D P=9,1)$; as principais frequências de estado civil foram de $65,17 \%$ casados/união estável, seguido de $26,35 \%$ solteiros. Em relação à escolaridade, $47,79 \%$ possuem pós-graduação latu sensu, $28,10 \%$ com ensino superior, $19,27 \%$ com pós- 
graduação stritu sensu, e 4,84 possuem ensino médio. Do ponto de vista profissional, $70,15 \%$ ocupam cargos de nível superior, além de que 62,65\% atuam na Esfera Federal, $36,72 \%$ na Distrital e 0,63\% em outras esferas. Em relação a participação nos treinamentos que levaram em consideração para responder a pesquisa, $84,72 \%$ indicaram que foi voluntária, enquanto $15,28 \%$ foram obrigados, além de que $67,27 \%$ afirmaram terem recebido orientação e apoio da chefia para participação em treinamentos. Após o retorno dos treinamentos, $59,15 \%$ consideraram estar satisfeitos com a execução do trabalho após colocar em prática o que foi aprendido.

\subsection{Procedimentos de Análise de Dados}

A análise textual consiste em um modal específico de análise de dados cujo objetivo é hierarquizar o conteúdo de textos produzidos a partir de diferentes fontes - tradicionalmente utilizadas nos estudos das Ciências Humanas Sociais - a fim de ampliar o entendimento por meio da percepção do relacionamento entre ideias, bem como da identificação das conclusões e das bases que as sustentam (Nascimento \& Menandro, 2006; Prestes, 2012).

Para Lahlou (2012), a análise textual, com uso da ferramenta qualitativa, permite conectar textos das abordagens científicas a partir de distribuições estatísticas e matemáticas, dessa forma, superando a dicotomia clássica entre quantitativo e qualitativo.

Para análise, foi utilizado o software Iramuteq 0.7 Alpha 2, com uso do Método de Classificação Hierárquica Descendente (CHD). A base de dados analisada é composta por respondentes localizados na unidade da Federação do Distrito Federal referente às potenciais dificuldades encontradas na aplicação do construto transferência de treinamento no contexto específico do setor público, traduzida em apenas uma das questões abertas do instrumento de pesquisa aplicado "Quais as principais dificuldades que você teve para colocar em prática no seu trabalho o aprendizado adquirido no treinamento?".

Ressalta-se que a escolha do uso do Iramuteq foi pelo fato de ser uma ferramenta gratuita que já possui dicionário de palavras em português, e que permite a análise textual de grande volume de palavras (Camargo \& Justo, 2013), o que é aplicável ao caso desta pesquisa devido ao tamanho da amostra de respondentes e corpus de palavras. Além disso, - Iramuteq permite a interpretação de resultados já processados com rigor científico, 
segurança e credibilidade, uma vez que realiza o processamento de dados qualitativos com análises estatísticas de textos (Souza et al., 2018).

\section{RESULTADOS}

Primeiramente, como ponto de partida das análises, elaborou-se, conforme Figura 1., nuvem de palavras a partir do córpus coletado para identificar a frequência das palavras que se destacaram nas respostas dos participantes da pesquisa. Pôde-se verificar que as palavras com mais relevância foram: "não" (freq 331), "falta" (freq 213), "trabalho" (freq 146), "dificuldade" (freq 114), "prático" (freq 104), "conhecimento" (freq 88), "curso" (freq 88), "sistema" (freq 79), "resistência" (freq 78), "ainda" (freq 67), "colega" (freq 56), "novo" (freq 56) e "mudança" (freq 53). Tais resultados apontam a tendência de duas linhas de repostas: uma ligada aos participantes que não encontraram dificuldades em aplicar o conhecimento adiquido; e, outra linha dos participantes que têm dificuldade para transferir os conhecimentos por questões práticas e resistência dos colegas à mudança no trabalho e a novos conhecimentos.

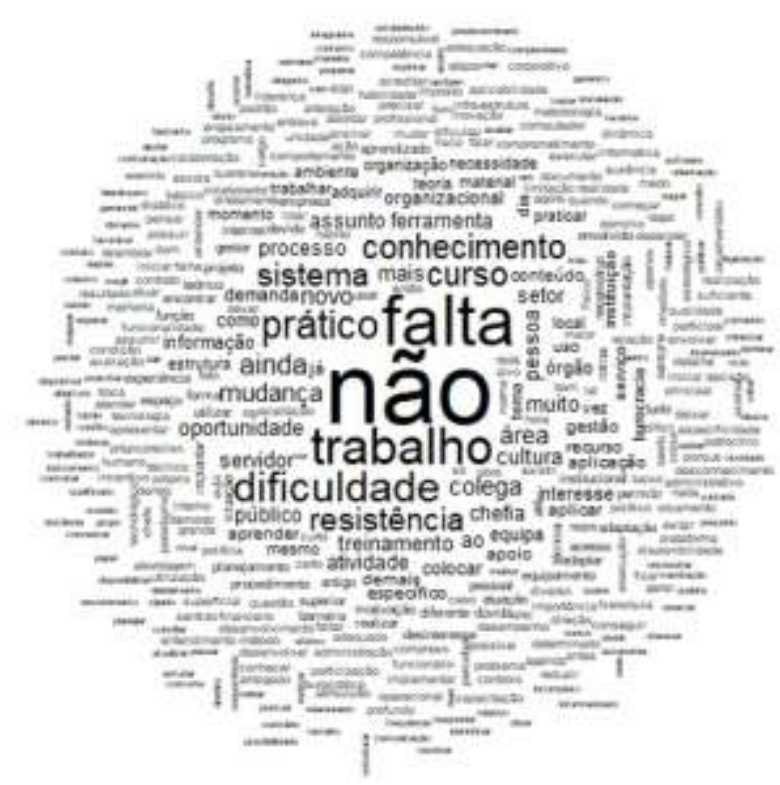

Figura 1. Nuvem de palavras gerada no IRAMUTEQ

O software analisou 1.433 segmentos de texto advindos da amostra de 1.427 participantes, em função da curta extensão da maioria das respostas. Foram geradas, a posteriori, três classes de análise, contendo 1036 segmentos de textos aproveitados. Esse extrato indica que $72,30 \%$ dos segmentos de texto foram utilizados na constituição das classes. A revisão 
de literatura utilizada apontou que uma retenção acima de $70 \%$ é considerada representativa na análise qualitativa.

O conteúdo das três classes apresentou as seguintes características: a) Classe 1, com 532 segmentos de texto $(51,35 \%)$, b) Classe 2 , com 180 segmentos de texto $(17,37 \%)$ e Classe 3 , com 324 segmentos de texto (31,27\%). Para ter uma melhor visualização das classes, elaborou-se a Figura 2 com a lista das principais palavras de cada classe geradas a partir do teste qui-quadrado. Nela surgem as evocações que apresentam vocabulário semelhante entre si e vocabulário diferente das outras classes. A seguir serão descritas, operacionalizadas e exemplificadas cada uma dessas classes emergidas da Classificação Hierárquica Descendente.

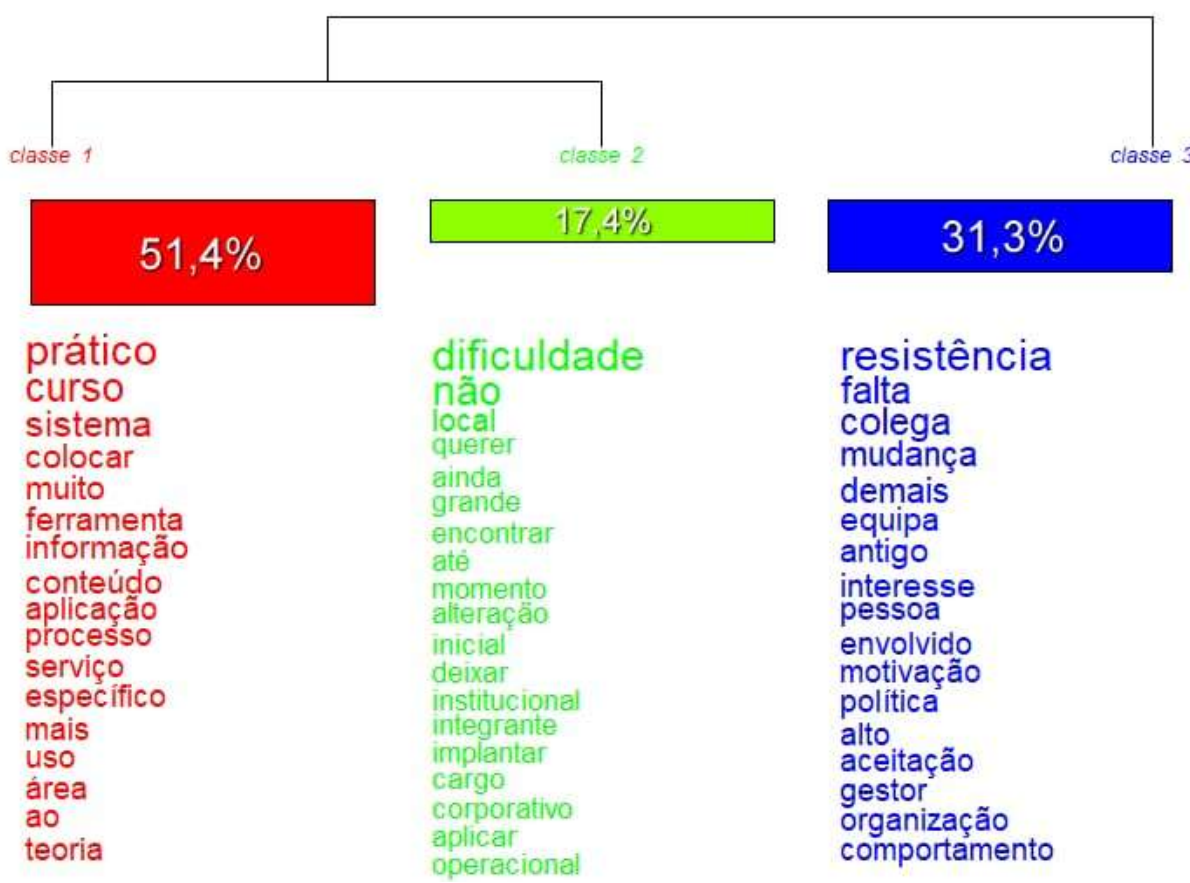

Figura 2. Classificação hierárquica independente gerada no IRAMUTEQ

A partir das expressões com qui-quadrado mais relevantes e o considerando o contexto empregado foram realizadas as denominações das classes da seguinte forma: Classe 1: Dificuldade de Aplicação Prática; Classe2: Inconsciência da Dificuldade; e, Classe 3: Resistência Cultural ao Novo.

A Classe 1 "Dificuldade de Aplicação Prática" sintetiza a falta de aderência entre o treinamento praticado e a realidade do contexto do trabalho do servidor público. Nesse caso 
a transferência é impedida pelo não alinhamento entre as competências desenvolvidas e o desempenho esperado na atuação do serviço público. A resposta a seguir é um exemplo relevante desta descrição: "A principal dificuldade está relacionada ao pouco uso do sistema de informações na minha rotina de trabalho pois o conhecimento adquirido no curso não está diretamente relacionado à atividade que desempenho" (respondente - Homem, 35 anos, Solteiro, Especialização, cargo nível superior, não é gestor, tempo de empresa 1 a 5 anos).

A primeira classe remete a problemas de oportunidade de execução da dimensão contextual de ambiente de trabalho (Baldwin, Ford, \& Blume, 2017; Ford, Baldwin, \& Prasad, 2018). Além disso, o fato dos servidores estarem participando de treinamentos que não são atrelados ao trabalho que irão desempenhar no dia-a-dia pode remeter a um problema de planejamento das ações de treinamento. Sendo assim, este resultado do levantamento das dificuldades permite reflexão das organizações públicas no sentido de repensar a forma que definem o público que irá participar dos treinamentos, ou seja, este resultado realmente pode contribuir com o planejamento das iniciativas de treinamento adequadamente, conforme indicado por Ford, Baldwin e Prasad (2018).

A Classe 2 "Inconsciência da Dificuldade" denota que ainda não houve tempo para o servidor ter a consciência da ausência ou presença de dificuldades na transferência de treinamento. Esse córpus trata que a transferência ainda é obscura, não indicando a ausência de dificultade devido à presença de facilidade, mas sim, há um desconhecimento se de fato, haverá êxito na transferência do treinamento para a rotina do serviço público. Como exemplo, tem-se a resposta “...a prática ainda é inicial portanto as dificuldades não estão mapeadas" (respondente - Homem, 48 anos, Casado/união estável, Especialização, cargo nível superior, gestor, tempo de empresa 1 a 5 anos). Da mesma forma, esta classe remete a ausência de oportunidade do uso do treinamento que influencia na transferência para o trabalho, conforme apontado por Baldwin, Ford e Blume (2017) e Ford, Baldwin e Prasad (2018).

A Classe 3 "Resistência Cultural ao Novo" contempla a falta de abertura à mudança por parte dos servidores públicos, seja na ação individual ou em equipe, em alterar o status-quo por meio da transferência. Essa significativa classe, aponta a presença de uma cultura da não-mudança, seja pela falta de domínio do novo ou pela insegurança na adoção de novas práticas de gestão e/ou padrões de trabalho. A resposta a seguir exemplifica a Classe 3: “... 
mudar a forma de abordagem, pois muitos colegas apresentavam resistência por falta de domínio sobre o assunto". (respondente - Homem, 37 anos, Casado/união estável, Especialização, cargo nível médio, não é gestor, tempo de empresa 1 a 5 anos).

A terceira classe permite agregar um novo aspecto do conexto do serviço público para a dimensão de ambiente de trabalho para transferência, considerando a consolidação da literatura proposta por Ford, Baldwin e Prasad (2018), que poderia passar a incluir também a variável de cultura organizacional. Apesar dos relatos trazerem aspectos de suporte de colegas e de gestores e das próprias características dos indivíduos sobre suas inseguranças ou falta de domínio, que são aspectos tratados pelos referidos autores, foi possível evidenciar o aspecto da cultura organizacional em si.

\section{CONCLUSÕES}

A análise textual com uso do Iramuteq nesta pesquisa permitiu, a partir de um grande volume de dados, ampliar o entendimento sobre o tema pesquisa, conforme recomendações da literatura (Camargo \& Justo, 2013; Nascimento \& Menandro, 2006; Prestes, 2012). Sendo assim, permitiu identificar as potenciais e reais dificuldades na transferência de treinamento no setor público, contribuindo com pesquisas recomendadas por Ford, Baldwin \& Prasad (2018) e Antunes, Nascimento, \& Bates (2018).

O uso de Softwares de Análise de Dados Qualitativos (SADQ) tem se mostrado cada vez mais presente no campo das Ciências Sociais Aplicadas (Camargo \& Justo, 2013), vide o portal de periódicos CAPES que apresenta mais de 2.500 artigos e capítulos de livros sobre o tema publicados nos últimos 5 anos <disponível em www.periódicos.capes.gov.br>.

Cumpre ressaltar que o emprego de Softwares de Análise de Dados Qualitativos (SADQ) não tem o intuito de dar à pesquisa qualitativa um enfoque puramente quantitativo, mas visa potencializar seu alcance (Camargo \& Justo 2013). O uso de SADQ permite analisar bancos de dados maiores e mais complexos, além de fornecer mais subsídios ao pesquisador para explorar, descrever e compara dados. No entanto, o software não é o método. Da mesma forma, as saídas de processamento dos softwares não correspondem a análise textual concluída (Lahlou 2012), pois o software é apenas um instrumento para exploração de dados e a interpretação deve realizada pelo pesquisador usando seu conhecimento contextual para fundamentar o fenômeno. 
Nesse contexto, a análise textual das respostas obtidas da amostra de participantes da presente pesquisa, para a questão "Quais as principais dificuldades que você teve para colocar em prática no seu trabalho o aprendizado adquirido no treinamento?", revelou a existência de variáveis inibidoras da transferência de treinamento na ambiência do setor público do Distrito Federal do Brasil. O conteúdo analisado foi agrupado em 3 (três) classes.

A primeira classe de conteúdo aponta para a dificuldade de aplicação prática entre o treinamento praticado e a realidade do contexto do serviço público do Distrito Federal. Nesse caso, a adversidade está relacionada inaplicabilidade do conteúdo estudado às atividades desempenhadas do servidor público. A segunda classe de conteúdo indica que o servidor ainda desconhece as possíveis dificuldades a serem enfrentadas na transferência do treinamento para a rotina do serviço público, dada a falta de tempo hábil para avaliá-las. Esse corpus trata que a transferência ainda é obscura, não indicando a ausência de dificuldade devido à presença de facilidade, mas sim, há um desconhecimento se de fato, haverá êxito na transferência do treinamento para a rotina do serviço público. A última classe evidencia que no ambiente de serviço público do Distrito Federal há, em alguns casos, resistência às mudanças do status-quo, seja na ação individual dos servidores ou em equipe. Essa classe comprova a presença de uma cultura da não-mudança, seja pela falta de domínio do novo ou pela insegurança na adoção de novas práticas de gestão ou padrões de trabalho.

A limitação desta pesquisa é decorrente do uso de pergunta aberta em questionário eletrônico, uma vez que as respostas são mais diretas e objetivas, sem a possibilidade do pesquisador aprofundar mais a percepção do participante. No entanto, este fato não prejudica o resultado da pesquisa, uma vez que a intenção do tipo de coleta utilizada era obter percepções gerais de maior número de servidores públicos. Outra possível limitação do uso do software trata do risco de "falha" na interpretação e agrupamento de palavras que podem ser escritas da mesma forma, mas dependendo do contexto terem significados diferentes, como é o caso da palavra "para" que pode ser a conjugação do verbo parar ou uma preposição.

Do ponto de vista metodológico, sugerem-se as seguintes pesquisas: (i) estudos comparados com diferentes técnicas de análise de dados, sejam diferentes softwares e até mesmo entre software e análises diretas pelos pesquisadores (ex: com análise categorial temática) para verificar a qualidade dos resultados gerados; (ii) outros tipos de análises que 
são permitiadas pelos sotwares de análise qualitativa (ex: análise de similitude); (iii) análises comparadas por perfil do respondente (ex: sexo, cargo, esfera de governo).

Em relação ao tema da pesquisa em si de transferência de treinamento, recomenda-se pesquisas longitudinais de análise do contexto com a aplicação dos treinamentos, verificar a existência de relações entre o tipo de participação (obrigatória ou voluntária) com a existência das dificuldades de implantação; aprofundar as possíveis causas das dificuldades quanto ao motivo da participação em treinamentos atrelados a planos de capacitações institucionais ou não.

Agradecimentos. À Escola Nacional de Administração Pública pela parceria que contribui para obtenção de uma amostra significativa à pesquisa.

\section{REFERÊNCIAS}

Abbad, G. S., Lacerda, E. R. M., \& Pilati, R. (2012). Medidas de motivação e valor instrumental do treinamento. In: Abbad, G. S., Mourão, L., Meneses, P. P. M., Zerbini, T., Borges-Andrade, J. E., Vilas-Boas, R. (org). Medidas de Avaliação em Treinamento, Desenvolvimento e Educação: ferramentas para gestão de pessoas. (p. 198-215). Porto Alegre: Artmed.

Abdullah Al-Swidi, Mohammed Al Yahya, (2017). Training transfer intention and training effectiveness: Assessing the gender differences using multi-group structural equation modelling approach, International Journal of Organizational Analysis, 25(5), 839-860, https://doi.org/10.1108/IJOA-07-2016-1043.

Antunes, A., Nascimento, J. L., \& Bates, R. A. (2018). The revised learning transfer system inventory in Portugal. International Journal of Training and Development, 22(4), 301-333.

Baldwin, T.T. \& Ford, J.K. (1988). Transfer of training: A review and directions for future research. Personnel Psychology, 41(1), 63-105.

Baldwin, T. T., Ford, J. K., \& Blume,B. D. (2017). The state of transfer of training research: moving toward more consumer-centric inquiry. Human Resource Development Quarterly, 1-12, DOI: 10.1002/hrdq.21278.

Bell, B. S., Tannenbaum, S. I., Ford, J. K., Noe, R. A., \& Kraiger, K. (2017). 100 Years of Training and Development Research: What We Know and Where We Should Go. Journal of Applied Psychology, 1-19, http://dx.doi.org/10.1037/apl0000142.

Blume, B. D., Ford, J. K., Baldwin, T. T., \& Huang, J. L. (2010). Transfer of training: a meta-analytic review. Journal of Management, 36(4), 1065-1105.

Camargo, B. V., \& Justo, A. M. (2013). Iramuteq: um software gratuito para análise de dados textuais. Temas em Psicologia, 21(2), 513-518.

Ellington , J. K. , Surface , E. A. , Blume , B. D. , \& Wilson , M. A. ( 2015 ). Foreign language training transfer: Individual and contextual predictors of skill maintenance and generalization. Military Psychology , 27,36 - 51 . htpp://dx.doi.org/10.1037/mil0000064.

Ford, J.K., Baldwin, T.P., \& Prasad J. (2018). Transfer of training: the known and the unknown. Annual Review of Organizational Psychology and Organizational Behavior, 5, 201-225. 
Hughes, A. M., Zajac, A., Spencer, J. M., Salas, E. (2018). A checklist for facilitating training transfer in organizations. Journal of training and development, 1-12. doi: 10.1111/ijtd.12141.

Lahlou, S. (2012). Text mining methods: An answer to Chartier and Meunier. Papers on Social Representations, 20(38), 1-7.

Nascimento, A. R. A., \& Menandro, P. R. M. (2006). Análise lexical e análise de conteúdo: Uma proposta de utilização conjugada. Estudos e Pesquisas em Psicologia, 6(2), 72-88.

Ranzini, M.S., \& Bryan, N.A.P. (2017). Capacitação e formação para o setor público e os modelos de escola de governo no Brasil. Revista do Serviço Público, 68(2), 417-438.

Rodrigues, A. C. A. L., Petine, E. T. P. D. S., \& Rodrigues, F. G. (2020). Capacitação Estratégica com Readequação Orçamentária para Melhoria do Serviço Público em uma Instituição Federal de Ensino Superior. Revista Gestão \& Tecnologia, 20(1), 278-304. http://dx.doi.org/10.20397/2177$\underline{6652 / 2020 . v 20 i 1.1729 .}$

Souza, M. A. R., Wall, M. L., Thuler, A. C. M. C., Lowen, I. M. V., \& Peres, A. M. (2018). O uso do software IRAMUTEQ na análise de dados em pesquisas qualitativas. Revista da Escola de Enfermagem da USP, 52(e03353), 1-7. 